10.2478/jbcr-2021-0O1O

Case Report

\title{
A RARE CLINICAL CASE OF LIPOMA IN THE SOFT TISSUES OF THE SUBLINGUAL SPACE: A CASE REPORT
}

\section{Petya G. Kanazirska, Mery A. Hristamyan-Cilev', Nikolay D. Kanazirski ${ }^{2}$}

\author{
Department of Imaging Diagnostics, \\ Dental Allergology and Physical \\ Therapy, \\ Faculty of Dental Medicine, $M U$ - \\ Plovdiv \\ ${ }^{1}$ Department of Epidemiology and \\ Disaster Medicine, \\ Faculty of Public Health, MU - Plovdiv \\ ${ }^{2}$ Department of Oral Surgery, \\ Faculty of Dental Medicine, $M U$ - \\ Plovdiv
}

\author{
Corresponding Author: \\ Petya G. Hadzhigeorgieva-Kanazirska, \\ Department of Imaging diagnostics, dental \\ allergology and physical therapy, \\ Medical University of Plovdiv, Faculty of \\ Dental Medicine, \\ 3, Hr. Botev Str. \\ Plovdiv, 4000 \\ Bulgaria \\ e-mail: petyao182@abv.bg
}

Received: February 10, 2020

Revision received: June 11, 2020

Accepted: April 22, 2021

\section{Summary}

This report presents clinical case with a histopathologically established lipoma of the sublingual space. Lipomas are benign mesenchymal neoplasms produced by adipocytes. Our search in the literature demonstrated that the development of lipomas in the sublingual space is extremely rare. In the oral cavity, it is seen in less than $5 \%$ of patients. In this paper, we describe clinical features, imaging diagnostic and histopathological features. Lipomas are characterized by slow growth and could be asymptomic, therefore diagnosis could be delayed.

This report describes and discusses the case of a 64 yearsold-man with a formation, located below the left tongue, for a period of 4 years. The patient had repeatedly consulted with Ear-Nose-Throat (ENT) specialists and dentists who recommended surgical treatment. During the last month of the monitoring, the formation had grown significantly and the chewing and speech function had gradually worsened.

Keywords: adipocytes, lipoma, submandibular space

\section{Introduction}

The prevalence of lipomas in the submandibular space is very limited - academic studies report it in only $1 \%$ to $5 \%$ of cases. There are three types of non-lithoma classification known in medical practice: parosteal lipomas, occurring on the surfaces of the bone; deep lipomas - occurring in deep soft tissue; and superfial lipomas, found in the subcutaneous tissue. Approximately $13 \%$ of the lipomas are located in the head and neck [1]. A lipoma might contain elements other than adipose tissue, such as blood vessels, muscle fibers, fibroblastic and bone tissue. Imaging and histopathological examination could help establishing the diagnosis, although lipomas are usually diagnosed by clinical examination [2]. Taking into consideration the fact that the formation is located in the soft tissue, diagnostic-imaging examination with the highest informative value is ultrasonography. The histopathologycal examination is important to make a diagnosis. 


\section{Case report}

The case examined is of a 64-year-old male patient with a formation located below the left tongue with a history of 4-year monitoring. The patient had repeatedly consulted with an EarNose-Throat (ENT) specialists and dentists who recommended surgical treatment. He reported that over the years the formation had been changing in size and volume. The alteration of the oral area had increased significantly during the month before the examination and he had difficulties chewing and speaking.

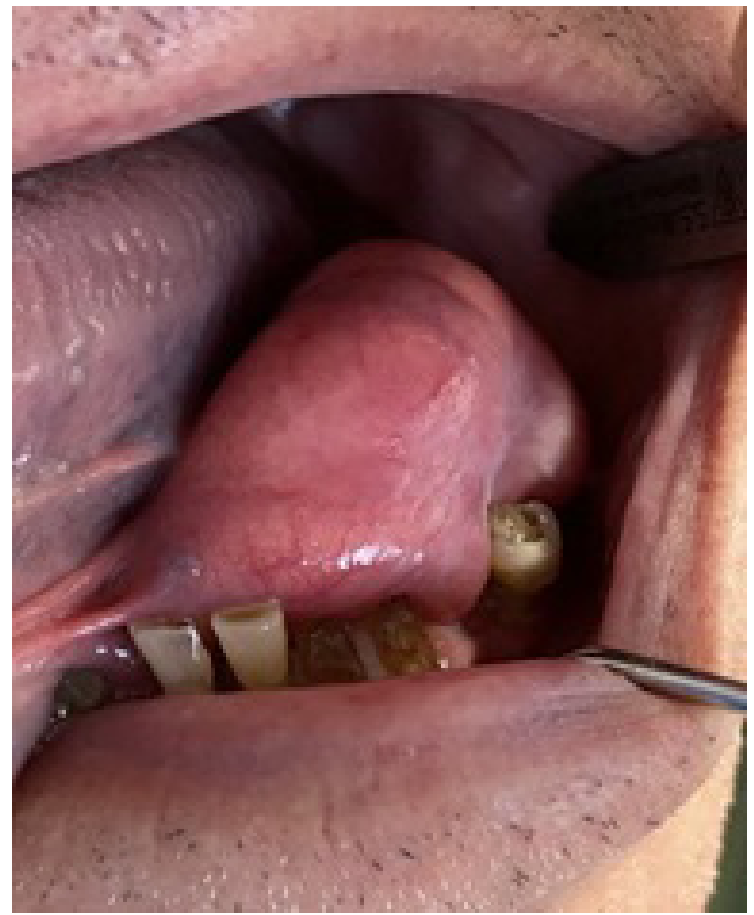

Figure 1

Upon admission, an ultrasonography was performed. The examination revealed a relatively hyperechogenic mass which was well-circumscribed by the adjacent muscles. No connection was found between the process and the underlying submandibular duct. Surgery was performed under local anestesia with sedation. After preparation of the mucosa, the formation under the tongue was revealed. Intraoperatively, it was also found that the formation was not related to the ductus of the left submandibular gland (sialoadenectomy totalis), and removal was not necessary. The formation was easily removed from the surrounding tissue, as could be seen from the cut mass shown in Figure 2. The terminal mandibular nerve was well-preserved and the other glands were not involved.
According to the histopathological examination of the sample, adipocytes were identified in lobules separated by muscle bundles and fibro-connective tissue. These characteristics proved the dignosis of lipoma, made by the attending physician.

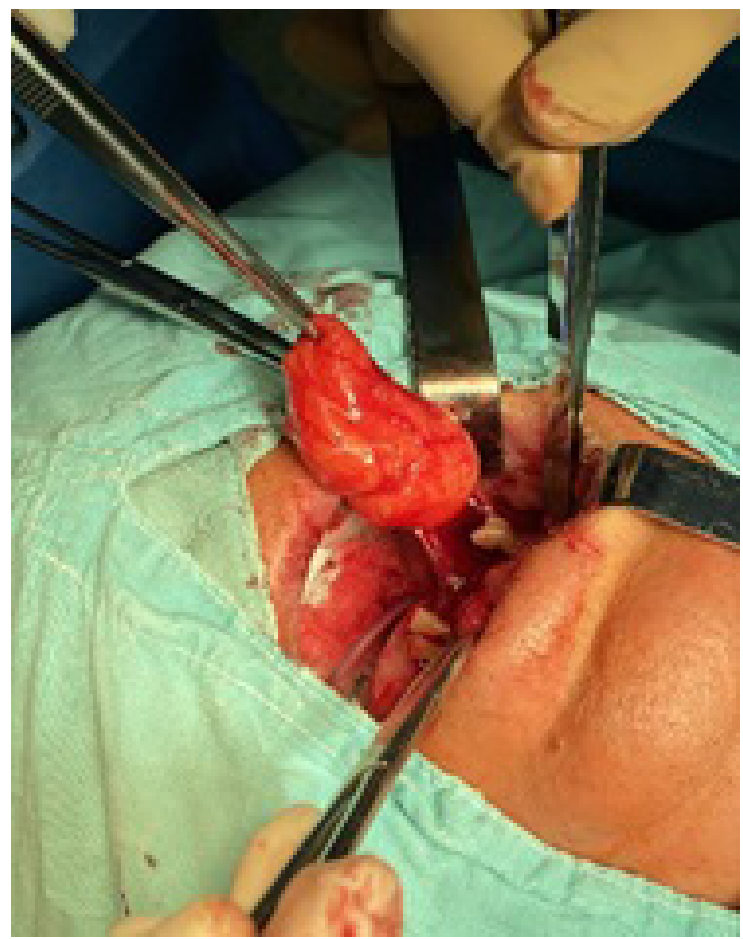

Figure 2

\section{Discussion}

Lipomas should be considered as one of the differential diagnoses of neck masses, although they are relatively rare in the head and neck areas. The annual incidence of lipoma is 1 in 1000 individuals [2]. The different variants of this condition are lipomatosis, angiolipomas, chondroidolipomas, pleomorphic lipomas, lipoblastomas, spindle-cell lipomas, and lipoblastomatosis. Due to the proliferation of chemically and histologically normal adipose tissue, cases of solitary lipoma have been observed as a common soft-tissue tumor. By its very nature, solitary lipoma is not considered a hamartoma because it has no development in origin, but is rather regarded as having a true neoplasm. Intraoperatively, lipomas could be considered as soft, smooth, motile, yellow, shiny, encapsulated formations. Occasionally, they also occur as lobulated subcutaneous masses. The lesions in the microscopic examination show a capsule of fibrosus and central vacuole 
or lobular growth of mature adipocytes with demarcated borders.

All patients should be informed of the risks and advised to contact their periodontologist or oral surgeon immediately, if they notice any pain or swelling of the jaws, loose teeth, pus discharge, bad taste or numbness or any unusual sensation in the mouth [6].

In some cases, lipomas are also likely to penetrate the adjacent muscles. In such cases, the presence of infiltrative lipomas is to be confirmed. Their origin might be related to a number of syndromes, such as Gardner's syndrome, Madelung's disease, hereditary multiple lipomatosis, and Decrum's disease. Adachi et al. [3] and Pusiol et al. have reported cases of oncocytic sialolipoma of the submandibular gland and deep intramuscular lipomas in the submandibular region [1]. Other researchers, such as Gultekin [4], have reported a single case of parosteal lipoma. In their study, Furlong et al. reported that lipomas in the head and neck are common in the parotid region followed by buccal mucosa and lip [5]. Sometimes a clinical examination alone is not enough to identify the nature and exact location of the mass and make a diagnosis. In such cases, the use of histopathological examination and imaging is extremely useful. Magnetic resonance imaging and ultrasound can differentiate lipoma from other soft tissue tumors.

Ultrasonography and histopathological examination helped diagnose the case. No decision was made to remove neither the sublingual nor submandibular left glands (sialoadectomy totalis). The prognosis of the patient's superficial lipoma is good and the risk of relapse is low.

\section{Conclusion}

Although lipomas are relatively rare in the maxillo-facial area, they should be considered as one of the differential diagnoses. Solitary lipoma is a soft-tissue tumor resulting from proliferation of histologically and chemically normal adipose tissue. Solitary lipoma has no developmental origin and is therefore not considered as hamartoma, but rather as a true neoplasm. Clinical examination alone is not sufficient to identify the nature and exact location of the mass. Imaging and histopathological examination may be useful in such a situation. The prognosis for superficial lipoma is good and the risk of reccurences is low.

\section{References}

1. Pusiol T, Franceschetti I, Scialpi M, Piscioli I. Oncocytic sialolipoma of the submandibular gland with sebaceous differentiation: A new pathological entity. Indian J Pathol Microbiol 2009;52:379-82.

2. Tsolov R, Firkova E, Chenchev I, Jordanov G. Paecheva, S. Bisphosphonate-related osteonecrosis of the jaw a 3-years retrospective study of frequency and risk factors. J of IMAB, 2019; 25(3), 2617-21.

3. Adachi P, Kaba SP, Soubhia AM, Shinohara EH. Intermuscular lipoma of the submandibular space. Indian J Dent Res 2011;22:871-2.

4. Gultekin SE, Kahraman S, Karady K. Parosteal osteochondrolipoma of the mandible. J Oral Maxillofac Pathol 2012;16:280-2.

5. Furlong MA, Fanburg-Smith JC, Childers EL. Lipoma of the oral and maxillofacial region: Site and subclassifi cation of 125 cases. Oral Surg Oral Med Oral Pathol Oral Radiol Endod, 2004;98:441-50.

6. Tsolov R, Firkova E, Yordanov G. Advanced stage 3 medication-related osteonecrosis of the mandible in a cancer patient on denosumab therapy (A case report), Biocell, 2019; 43 (51):240-4. 